\title{
Bone-sparing effects of rituximab and body composition analysis in a cohort of postmenopausal women affected by rheumatoid arthritis - retrospective study
}

\author{
Suhel Gabriele Al Khayyat ID, Paolo Falsetti ID, Edoardo Conticini ID, Roberto D'Alessandro ID, \\ Francesca Bellisai, Stefano Gentileschi ID , Caterina Baldi ID , Marco Bardelli, Luca Cantarini ID, \\ Bruno Frediani ID \\ Rheumatology Unit, University Hospital of Siena, Italy
}

\begin{abstract}
Objective: Osteoporosis is the most common bone tissue disease and it is characterized by a reduced bone mineral density (BMD). The main physiopathological mechanisms converge on the uncoupling between bone formation and resorption, thus leading to an enhanced risk of fractures. Several papers have documented the inverse relationships linking high inflammatory cytokines, anticitrullinated protein antibodies, rheumatoid factor, and BMD in rheumatoid arthritis (RA). Rituximab (RTX) is a chimeric monoclonal antibody directed against the CD20 receptor of B cells. Since the Food and Drug Administration approved it for RA in 2006, there have been many clinical experiences regarding its use. Nevertheless, few studies evaluate the effect of rituximab on BMD. RA is a disease characterized by immune dysfunction with high levels of inflammatory cytokines, autoantibodies, and it is reasonable that a B cell depleting therapy could restore a physiological cytokine balance, thus exerting an osteoprotective effect on the bone tissue. The purpose of this paper is to highlight any difference in BMD and to assess differences in body composition over a retrospective 18-month follow-up period after RTX treatment with a B cell depleting therapy.

Material and methods: We analyzed by dual energy X-ray absorptiometry BMD expressed as $\mathrm{g} / \mathrm{cm}^{2}$ and body composition modifications over 18 months with RTX treatment of 20 postmenopausal RA patients.

Results: After eighteen months of therapy with RTX, a statistically significant increase in vertebral (L1-L4) BMD and the stability of femoral BMD were documented.

Conclusions: Rituximab is associated with an improvement of vertebral and preservation of femoral BMD, suggesting a bone-sparing effect due to B cell depletion. Furthermore, patients displayed a redistribution of fat masses toward the hip region.

Key words: bone, rituximab, osteoporosis, rheumatoid arthritis.
\end{abstract}

\section{Introduction}

Rheumatoid arthritis (RA) is defined by the latest American College of Rheumatology/European League Against Rheumatism (ACR/EULAR) classification criteria [1] as a chronic and systemic disease in which immunologically mediated inflammation of synovial joints may lead to destruction of articular structures. Osteoporosis is the most common bone tissue disease where pathophysiological mechanisms involve an uncoupling between bone formation and resorption, thus leading to a reduction of bone mineral density (BMD) and to an increased risk of fractures [2]. Bone homoeostasis is

Address for correspondence:

Suhel Gabriele Al Khayyat, University Hospital of Siena, Viale Mario Bracci, 1653100 Siena, Italy, e-mail: alkhayyatsuhelg@gmail.com Submitted: 10.02.2021; Accepted: 06.08.2021 
regulated by the WNT pathway, an elegant (as much as complex) transcellular signaling mechanism that regulates bone formation, whose alteration has been linked to a higher number of erosions and lower bone mineral density in RA [3].

Recent papers have documented that an alteration of the cytokine pool in a pro-inflammatory sense in RA causes an imbalance of WNT signaling due to the direct effect of tumor necrosis factor $\alpha$ (TNF- $\alpha$ ), interleukin 6 (IL-6) and interleukin 1 (IL-1) on osteoclasts, favoring their maturation and differentiation [4]. Furthermore, it seems that anti-citrullinated peptide antibodies (ACPA), as well as rheumatoid factor (RF), play a pathogenetic role directly activating osteoclast-mediated resorption via key osteoclastogenic cytokine receptor activator of $N F-\kappa B$ ligand (RANKL) induction [5], thus leading to joint erosions and osteoporosis. Moreover, recent evidence demonstrated the role of activated B lymphocytes [6] in favoring loss of mineralization in RA. In addition, an altered body composition too may represent the result of a cytokine pool imbalance toward an inflammatory state [7, 8]. For these reasons, we aimed to investigate retrospectively the effects of rituximab (RTX) on bone and body composition in a cohort of postmenopausal rheumatoid arthritis patients.

\section{Material and methods}

We studied vertebral, whole femoral and neck femoral BMD and body composition using dual energy X-ray absorptiometry (DXA, Lunar prodigy version 1.72) of 20 postmenopausal women affected by RA according to ACR/EULAR criteria before and after 18 months of treatment with RTX [1]. Quality control of the device was performed every day. RTX was administered with a loading dose of two infusions of $500 \mathrm{mg}$ or $1 \mathrm{~g}$ depending on patients' weight during the first two weeks of treatment with a premedication of $100 \mathrm{mg}$ of methylprednisolone and $1000 \mathrm{mg}$ of acetaminophen; subsequently infusions were scheduled every six months. At the end of the study each patient underwent eight RTX infusion sessions and reached a methylprednisolone cumulative dose of $800 \mathrm{mg}$. Medical history, weight, height and body mass index (BMI) were collected.

Diagnosis of osteoporosis was performed when the T-score of patients deviated below -2.5 from the standard deviation compared with the reference population according to World Health Organization criteria. None of the considered subjects had vertebral or femoral osteoporosis at baseline. Also none of the RA patients included in the analysis had a history of chronic obstructive pulmonary disease (COPD), chronic heart failure (CHF) or chronic kidney disease (CKD). Body composition of RA patients was analyzed before and after treatment with
RTX, using themselves as controls. Appendicular lean mass (ALM, $\mathrm{kg} / \mathrm{m}^{2}$ ) was calculated as the sum of fat-free mass minus bone mineral content of lower and upper limbs. Skeletal muscle mass index $\left(\mathrm{SMI}, \mathrm{kg} / \mathrm{m}^{2}\right)$ was calculated as ALM divided by height squared according to Baumgartner's criteria [7]. A low muscle mass was identified for women when found 2 SD below the mean value of young adults (female: $<5.5 \mathrm{~kg} / \mathrm{m}^{2}$ ).

Ethical standards - the presented retrospective work is based on data derived from existing medical records and did not require additional consent, all patients agreed to the diagnostics and procedures performed.

\section{Characteristics of the studied group}

Of the 20 studied patients, $n=3$ had Hashimoto's thyroiditis under levothyroxine treatment, $n=3$ had hypertension, $n=1$ had a history of bladder cancer and $n=1$ had hypercholesterolemia. Among all studied patients $n=7$ were treated with glucocorticosteroids (GCs) with a low dose of prednisone ( $<7.5 \mathrm{mg} /$ day), and only $n=4$ patients were taking oral vitamin D. Mean ACPA and RF were available from our records for 10 patients: respectively $165 \mathrm{IU} / \mathrm{I}( \pm 130)$ and $126 \mathrm{UI} / \mathrm{I}( \pm 105)$. Mean disease duration at baseline was 11.86 years $( \pm 10.8)$. Clinical characteristics of studied patients are summarized in Table I.

\section{Statistical analysis}

Statistical analysis was performed using GraphPad Prism version 8.0.0 for Windows, GraphPad Software, San Diego, California USA (www.graphpad.com).

Demographic data were analyzed, and each variable expressed as mean plus standard deviation using descriptive statistics. Inferential statistical tests were used to compare means of each region previously ana-

Table I. Epidemiological characteristics and anthropometrics measures of rheumatoid arthritis (RA) patients

\begin{tabular}{|lc|}
\hline Parameters & RA patients \\
\hline Number & 20 \\
\hline Gender & F \\
\hline Age [years] & $65.07 \pm 12.90$ \\
\hline Weight $[\mathrm{kg}]$ & $67.29 \pm 14.27$ \\
\hline Height $[\mathrm{cm}]$ & $164 \pm 8.238$ \\
\hline BMI [kg/m²] & $24.96 \pm 4.555$ \\
\hline Disease duration [years] & $11 \pm 10.8$ \\
\hline ACPA levels [IU/I] & $130 \pm 165$ \\
\hline RF levels [IU/I] & $126 \pm 105$ \\
\hline
\end{tabular}

$B M I$ - body mass index, F-female, RA - rheumatoid arthritis, ACPA - anti-citrullinated protein antibodies, RF-rheumatoid factor, IU - international units. 
lyzed with DXA. Subsequently, we compared BMD of the lumbar spine, femoral neck, and whole femur with the $t$-test for paired observations if data followed a normal distribution, or using Wilcoxon's test for paired statistics when data deviated from a Gaussian distribution. The same tests were run to compare each variable derived from body composition analysis. Results were considered statistically significant when $p<0.05$.

\section{Results}

The results showed a significant increase in lumbar spine BMD (1.031 \pm 0.11 vs. $1.110 \pm 0.10, p<0.005)$, while no significant difference was found for femoral BMD. The data are shown in Figure 1 and Table II.

Erythrocyte sedimentation rate (ESR) expressed in millimeters/hour (available for only 13 matched comparisons) displayed a significant reduction from baseline (32 \pm 16 vs. $25 \pm 14, p<0.0205$ ).

Lean mass and fat mass values increased from baseline, although not significantly (ALM $16.21 \pm 3.60 \mathrm{vs}$. $17.84 \pm 4.03$; SMI $6.03 \pm 0.99$ vs. $6.42 \pm 1.85$ ns; body fat $\%$ $37.45 \pm 10.82$ vs. $40.19 \pm 8.44$ ns). The results are shown in Table III.

\section{Discussion}

The investigation highlighted a clear and significant increase of vertebral BMD and documented the stability of femoral mineralization in RA patients after 18 months of treatment with B cell depleting therapy. Obtained results may confirm the pathogenic role of activated $B$ cells in bone loss in RA, accordingly with what is reported in the literature [6].

Several papers demonstrated the active role of $B$ lymphocytes in modulating key osteoclastogenic cytokine RANKL and RANKL/osteoprotegerin (OPG) ratio, therefore actively intervening in bone homeostasis and being the bridge between immune and skeletal systems $[6,8,9]$.

Osteoprotegerin belongs to the superfamily of tumor necrosis factor receptors (TNFR), exists only in soluble form and can prevent bone resorption acting as a decoy receptor binding RANKL, thus being a bone savior.

Receptor activator for NF-KB ligand is also a part of the TNFR superfamily, but unlike OPG, which prevents bone loss, is the main actor of bone resorption by promoting osteoclast formation.
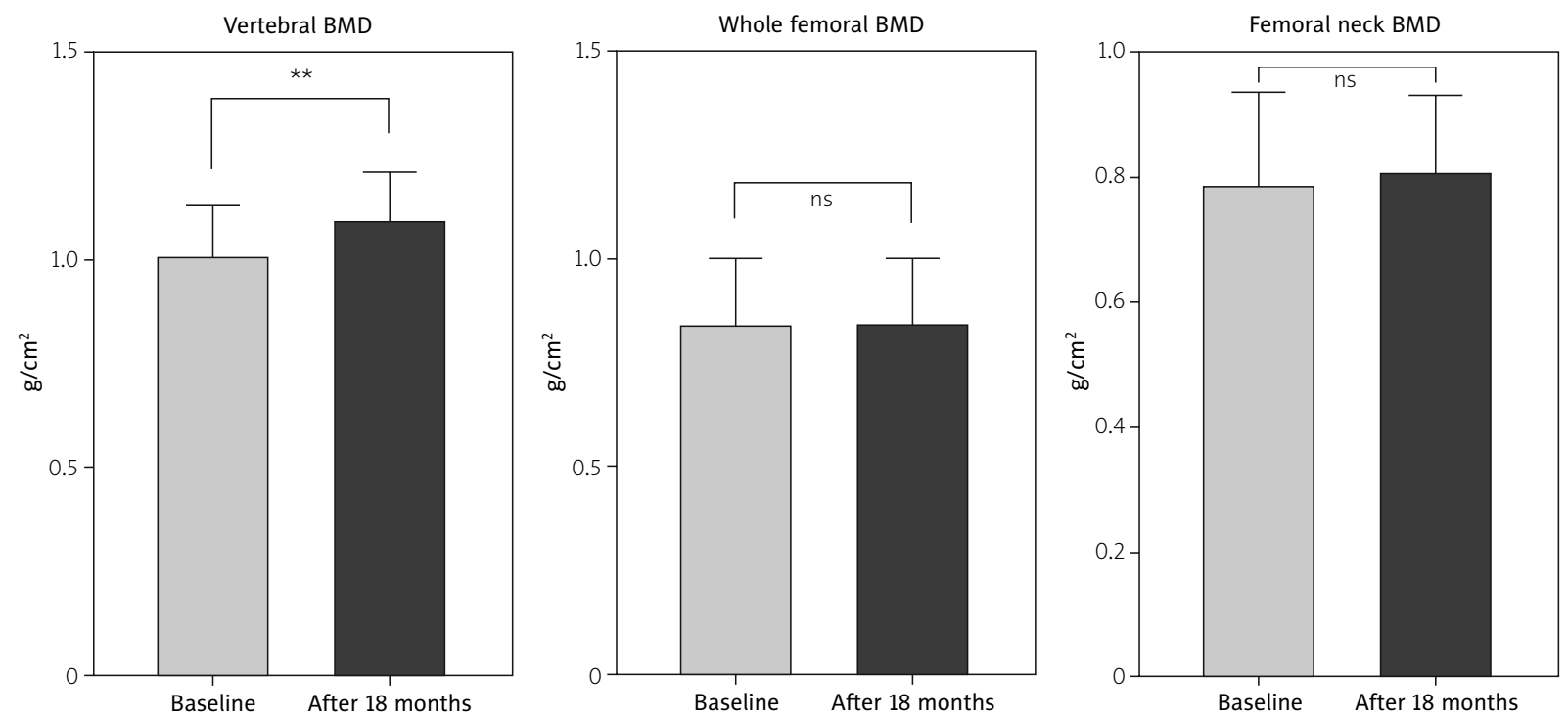

Fig. 1. Bone mineral density (BMD) modification after 18 months of therapy with rituximab.

Table II. Differences in bone mineral density (BMD) at baseline and after 18 months of rituximab (RTX) therapy

\begin{tabular}{|lccc|}
\hline BMD $\left[\mathrm{g} / \mathrm{cm}^{2}\right]$ & Baseline $(n=20)$ & After 18 months $(n=20)$ & $p$-value \\
\hline Lumbar spine (L1-L4) & $1.031 \pm 0.11$ & $1.110 \pm 0.10$ & $0.0029^{\text {** }}$ \\
\hline Whole femur & $0.848 \pm 0.15$ & $0.853 \pm 0.14$ & 0.622 (ns) \\
\hline Femur neck & $0.793 \pm 0.14$ & $0.808 \pm 0.12$ & 0.140 (ns) \\
\hline
\end{tabular}

Mean values of $B M D\left(\mathrm{~g} / \mathrm{cm}^{2}\right)$ at baseline and after 18 months therapy with rituximab, ${ }^{* *} p<0.005, n s$ - not statistically significant. 
Table III. Changes in body analysis composition at baseline and after 18 months of rituximab (RTX) therapy

\begin{tabular}{|lccc|}
\hline Body composition analysis & Baseline & After 18 months & $p$-value \\
\hline Total lean mass $[\mathrm{kg}]$ & $39.94 \pm 8.74$ & $38.64 \pm 8.19$ & 0.278 (ns) \\
\hline BF [\%] & $37.45 \pm 10.82$ & $40.19 \pm 8.44$ & $0.248(\mathrm{~ns})$ \\
\hline ALM [kg] & $16.21 \pm 3.60$ & $17.84 \pm 4.03$ & $0.476(\mathrm{~ns})$ \\
\hline SMI [kg/m²] & $6.03 \pm 0.99$ & $6.42 \pm 1.85$ & $0.445(\mathrm{~ns})$ \\
\hline Total fat mass [kg] & $25 \pm 10.45$ & $26.58 \pm 9.43$ & $0.382(\mathrm{~ns})$ \\
\hline Android fat [\%] & $43.07 \pm 13.33$ & $45.89 \pm 10.54$ & 0.320 (ns) \\
\hline Gynoid fat [\%] & $40.28 \pm 10.08$ & $43.02 \pm 10.65$ & 0.099 (ns) \\
\hline Android/gynoid fat ratio & $1.07 \pm 0.28$ & $1.10 \pm 0.31$ & 0.999 (ns) \\
\hline
\end{tabular}

Body composition features of RA patients before and after treatment with rituximab, BF-body fat, ALM - appendicular lean mass, SMI-skeletal muscle index.

B lymphocytes represent an important source of OPG, nevertheless during inflammatory circumstances OPG production decreases, shifting toward RANKL produced by $T$ lymphocytes activated by pro-inflammatory cytokines. The chronic inflammatory stimulus in arthritic patients plays a pivotal role in bone loss by promoting the shift toward RANKL production in B activated lymphocytes, thus altering the RANKL/OPG ratio and facilitating BMD reduction. A significant loss of bone mineralization attributable to an immunological dysfunction of activated $B$ cells is also described in multiple myeloma, human immunodeficiency virus (HIV) [8] and periodontal pathology [9]. Diseases very different from each other but united by RANKL/OPG ratio imbalance and by a high inflammatory load which may lead to the development of bone loss and osteoporosis.

Our results differ from those reported in other papers, where RTX therapy did not produce effects on vertebral BMD and indeed displayed bone loss at the femoral level [10]. Although these investigations were conducted longitudinally, the follow-up of patients did not exceed 12 months, a time probably insufficient to observe significant changes in BMD. None of our patients were osteoporotic at the beginning of the study and perhaps, starting from a lower degree of mineralization and a larger sample, an improvement in BMD could be observed at the femoral level too. Furthermore, increases of lumbar spine BMD and of muscle masses occurred in patients despite low dose GC therapy in 7 patients and 800 mg of methylprednisolone used as premedication during 18 months of therapy. Although the deleterious effects of GCs on bone [11] and muscle tissues [12] are known, a paper [13] demonstrated that a high dose GC pulse therapy did not lower BMD of RA patients during 18 months of follow-up.

Whether these observations are due to a direct effect of therapy on cytokine resources, or to an improvement in quality of life and in an increase of physical activity among RA individuals, remains to be investigated.
An altered body composition, such as an increase in percentage of fat mass, a reduction of muscle mass and a prevalent android fat distribution, in concert with an increased incidence of metabolic syndrome, is widely described in RA [14]. Several studies have demonstrated that blocking IL-6 [15] or TNF- $\alpha$ [16] produced an increase in the BMD in RA patients and improved their body composition [17]. Contrary to what we expected, RTX therapy did not cause any significant change in patients' muscle mass or fat mass representation. The anti-CD20 receptor can be expressed by other cell lines [18] and in RA there is reported an increase in circulating TNF- $\alpha$ as well as IL- 6 $[19,20]$. Many studies highlight the correlation between metabolic syndrome, increased inflammatory state, altered body composition and cardiovascular diseases [21]. An interesting paper reported that RTX may even lower IL-6 levels [12].

Based on these assumptions, it would have been expected that in our patients, alongside the preservation of BMD, the B cell depletion therapy produced a change in patients' body composition too.

Lack of this effect could be due to normal body fat (BF) percentage and muscle mass values displayed by most of our cases, allowing us to speculate that perhaps, in patients with an altered body composition at baseline, an improvement could also be observed in this regard as a consequence of a reduction in pro-inflammatory cytokine serum levels. There was an increase in gynoid masses, although not statistically significant.

Furthermore, an altered body composition [14, 22] and low bone mineral density are proven to share the same pathological mechanism of cardiovascular diseases [23], and this paper may prompt further research on the topic by highlighting the bone sparing effect in RA patients treated with RTX: an impairment of the RANKL/ $\mathrm{OPG}$ ratio is reported in correlation with arterial atherosclerosis [24] and in an increase of cardiovascular risk in RA [25], and thus RTX may play a cardioprotective effect 
in RA patients, although further investigations are needed to ascertain the validity of this hypothesis.

This study had several limitations due to its retrospective nature: it was not possible to further stratify patients according to their serum vitamin D levels, smoking habits, previous therapies and immunological status. Therefore, it will be necessary to assess prospectively and for an adequate time the effects of RTX on bone homeostasis and body composition.

\section{Conclusions}

Our study demonstrated that 20 non-osteoporotic RA patients had a significant increase in vertebral BMD and maintained their femoral BMD unchanged after 18 months of therapy with RTX. This evidence strengthens the hypothesis of bone sparing effects of RTX, despite a bolus of GCs for premedication prior to administration of this biological drug.

The authors declare no conflict of interest.

\section{References}

1. Aletaha D, Neogi T, Silman AJ, et al. 2010 Rheumatoid arthritis classification criteria: An American College of Rheumatology/ European League Against Rheumatism collaborative initiative. Arthritis Rheum 2010; 62: 2569-2581, DOI: 10.1002/art.27584.

2. Compston JE, McClung MR, Leslie WD. Osteoporosis. Lancet 2019; 393: 364-376. DOI: 10.1016/S0140-6736(18)32112-3.

3. Maruotti N, Corrado A, Cantatore FP, Cantatore FP. Osteoporosis and rheumatic diseases. Reumatismo 2014; 66: 125-135, DOI: 10.4081/reumatismo.2014.785.

4. Redlich K, Smolen JS. Inflammatory bone loss: Pathogenesis and therapeutic intervention. Nat Rev Drug Discov 2012; 11: 234-250, DOI: 10.1038/nrd3669.

5. Harre U, Georgess D, Bang H, et al. Induction of osteoclastogenesis and bone loss by human autoantibodies against citrullinated vimentin. J Clin Invest 2012; 122: 1791-1802, DOI: 10.1172/JCI60975.

6. Titanji K. Beyond antibodies: B cells and the OPG/RANK-RANKL pathway in health, non-HIV disease and HIV-induced bone loss. Front Immunol 2017; 8: 1851, DOI: 10.3389/fimmu. 2017.01851.

7. Baumgartner R, Koehler K, Gallagher D, et al. Epidemiology of sarcopenia among the elderly in New Mexico. Am J Epidemiol 1998; 147: 755-763, DOI: 10.1093/oxfordjournals.aje.a009520 [published erratum appears in Am J Epidemiol 1999; 149: 1161].

8. Titanji K, Vunnava A, Sheth AN, et al. Dysregulated B cell expression of RANKL and OPG correlates with loss of bone mineral density in HIV infection. PLoS Pathog 2014; 10: e1004497, DOI: 10.1371/journal.ppat.1004497.

9. Nikolajczyk BS. B cells as under-appreciated mediators of non-auto-immune inflammatory disease. Cytokine 2010; 50: 234-242, DOI: 10.1016/j.cyto.2010.02.022.

10. Wheater G, Elshahaly M, Naraghi K, et al. Changes in bone density and bone turnover in patients with rheumatoid ar- thritis treated with rituximab, results from an exploratory, prospective study. PLoS One 2018; 13: e0201527, DOI: 10.1371/ journal.pone.0201527.

11. Mitra R. Adverse effects of corticosteroids on bone metabolism: A review. PM R 2011; 3: 466-471, DOI: 10.1016/ j.pmrj.2011.02.017.

12. Jackman RW, Kandarian SC. The molecular basis of skeletal muscle atrophy. Am J Physiol Cell Physiol 2004; 287: C834-843, DOI: 10.1152/ajpcell.00579.2003.

13. Frediani B, Falsetti P, Bisogno S, et al. Effects of high dose methylprednisolone pulse therapy on bone mass and biochemical markers of bone metabolism in patients with active rheumatoid arthritis: A 12-month randomized prospective controlled study. J Rheumatol 2004; 31: 1083-1087.

14. Kerekes G, Nurmohamed MT, González-Gay MA, et al. Rheumatoid arthritis and metabolic syndrome. Nat Rev Rheumatol 2014; 10: 691-696, DOI: 10.1038/nrrheum.2014.121.

15. Chen YM, Chen HH, Huang WN, et al. Tocilizumab potentially prevents bone loss in patients with anticitrullinated protein antibody-positive rheumatoid arthritis. PLoS One 2017; 12: e0188454, DOI: 10.1371/journal.pone.0188454.

16. Zerbini CAF, Clark P, Mendez-Sanchez L, et al. Biologic therapies and bone loss in rheumatoid arthritis. Osteoporos Int 2017; 28: 429-446, DOI: 10.1007/s00198-016-3769-2.

17. Tournadre A, Pereira B, Dutheil F, et al. Changes in body composition and metabolic profile during interleukin 6 inhibition in rheumatoid arthritis. J Cachexia Sarcopenia Muscle 2017; 8: 639-646, DOI: 10.1002/jcsm.12189.

18. Alunno A, Carubbi F, Bistoni O, et al. Interleukin (IL)-17-producing pathogenic T lymphocytes co-express CD20 and are depleted by rituximab in primary Sjögren's syndrome: A pilot study. Clin Exp Immunol 2016; 184: 284-292, DOI: 10.1111/cei.12771.

19. Wei ST, Sun YH, Zong SH, Xiang YB. Serum levels of IL-6 and TNF- $\alpha$ may correlate with activity and severity of rheumatoid arthritis. Med Sci Monit 2015; 21: 4030-4038, DOI: 10.12659/ MSM.895116.

20. Barr TA, Shen P, Brown S, et al. B cell depletion therapy ameliorates autoimmune disease through ablation of IL-6-producing B cells. J Exp Med 2012; 209: 1001-1010, DOI: 10.1084/ jem.20111675.

21. Srikanthan K, Feyh A, Visweshwar H, et al. Systematic review of metabolic syndrome biomarkers: A panel for early detection, management, and risk stratification in the West Virginian population. Int J Med Sci 2016; 13: 25-38, DOI: 10.7150/ijms.13800.

22. Gremese E, Tolusso B, Gigante MR, Ferraccioli G. Obesity as a risk and severity factor in rheumatic diseases (autoimmune chronic inflammatory diseases). Front Immunol 2014; 5: 576, DOI: 10.3389/fimmu.2014.00576.

23. Lampropoulos CE, Papaioannou I, D'Cruz DP. Osteoporosis A risk factor for cardiovascular disease? Nat Rev Rheumatol 2012; 8: 587-598, DOI: 10.1038/nrrheum.2012.120.

24. Golledge J, McCann M, Mangan S, et al. Osteoprotegerin and osteopontin are expressed at high concentrations within symptomatic carotid atherosclerosis. Stroke 2004; 35: 16361641, DOI: 10.1161/01.STR.0000129790.00318.a3.

25. López-Mejias R, Ubilla B, Genre F, et al. Osteoprotegerin concentrations relate independently to established cardiovascular disease in rheumatoid arthritis. J Rheumatol 2015; 42: 39-45, DOI: 10.3899/jrheum.140690. 